\title{
Les défis de la sécurité sociale mexicaine face aux catastrophes socio-naturelles
}

\section{Gabriela Mendizábal Bermúdez}

\section{(2) OpenEdition}

1 Journals

\section{Édition électronique}

URL : https://journals.openedition.org/rdctss/1672

DOI : $10.4000 /$ rdctss. 1672

ISSN : 2262-9815

Éditeur

Centre de droit comparé du travail et de la sécurité sociale

\section{Édition imprimée}

Date de publication : 1 avril 2019

Pagination : 154-167

ISSN : 2117-4350

\section{Référence électronique}

Gabriela Mendizábal Bermúdez, «Les défis de la sécurité sociale mexicaine face aux catastrophes socio-naturelles », Revue de droit comparé du travail et de la sécurité sociale [En ligne], 1 | 2019, mis en ligne le 01 novembre 2021, consulté le 13 novembre 2021. URL : http://journals.openedition.org/ rdctss/1672; DOI : https://doi.org/10.4000/rdctss. 1672

\section{cc)}

Revue de droit comparé du travail et de la sécurité sociale est mise à disposition selon les termes de la Licence Creative Commons Attribution - Pas d'Utilisation Commerciale - Pas de Modification 4.0 International. 


\section{LES DÉFIS DE LA SÉCURITÉ SOCIALE MEXICAINE FACE AUX CATASTROPHES SOCIO-NATURELLES}

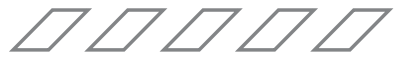

\section{RÉSUMÉ}

Les conséquences des catastrophes socio-naturelles posent de grands défis aux systèmes de sécurité sociale dans le monde. Cet article analyse et souligne l'intérêt légitime de répondre aux conséquences des catastrophes naturelles, comme c'est le cas dans certaines normes juridiques de la loi mexicaine sur la sécurité sociale qui, en cas de catastrophes naturelles, offre des avantages, la couverture santé entre autres, à la population en général et pas seulement aux bénéficiaires de l'ISSSTE (Institut national de sécurité sociale et de services sociaux des salariés). Cependant, il existe un grand besoin d'adapter la loi et d'étendre ainsi la couverture aux personnes vulnérables pour faire face à des événements naturels extrêmes.

MOTS CLÉS : Sécurité sociale, risques sociaux, catastrophes naturelles, protection sociale.

\section{ABSTRACT}

The consequences of socio-natural disasters impose great challenges to social security systems in the world. This paper analyses and underlines the legitimate interest in responding to the consequences of natural disasters, a fact that is clearly visible in certain legal standards of the Mexican Social Security Law, which in case of natural disasters provides benefits such as health care and others to all the population and not only to ISSSTE (State's Employees' Social Security and Social Services Institute) beneficiaries. However, there is a great need to adapt the Law and thus extend coverage to the most vulnerable in extreme natural events.

KEYWORDS : Social Security, Social Risks, Natural Disasters, Social Protection. 
es systèmes de sécurité sociale dans le monde ont évolué différemment dans chaque pays en fonction de facteurs tels que la culture, l'économie, l'histoire et la situation géographique, pour n'en citer que quelques-uns. Nonobstant, les orientations de la sécurité sociale sont toujours influencées par les besoins de la population qu'elle est censée protéger. Si la sécurité sociale a d'abord porté sur la protection des risques liés au travail ${ }^{1}$, elle a aujourd'hui pour objectif de protéger les membres d'une société des conséquences du changement climatique, et en particulier des catastrophes « naturelles ».

Il convient de noter que ces catastrophes, bien que naturelles, sont dans une large mesure le résultat des actions polluantes de l'homme et mettent en péril la viabilité financière de tous les systèmes de sécurité sociale dans le monde, en faisant émerger de nouveaux besoins et risques dans la société, dont l'augmentation de maladies comme le cancer de la peau suite à l'exposition aux UV du soleil, la destruction des habitations suite aux inondations ou la progression des pandémies, entre autres.

C'est pourquoi cet article porte sur la nécessité d'adapter et d'étendre la loi mexicaine pour protéger la population des défis inhérents aux catastrophes naturelles. Dans cette optique, suivant une méthodologique déductive, il s'agira d'exposer dans un premier temps les bases théoriques des termes « risque social » et « risque socio-naturel », « sécurité sociale » et " assurance sociale », avant de décrire brièvement les conséquences potentielles des catastrophes naturelles en matière de sécurité sociale (I). Dans un deuxième temps il s'agira de présenter une analyse des lois mexicaines en vigueur, en déterminant celles qui s'appliquent aux bénéficiaires de l'assurance sociale et celles qui s'appliquent à la population en général, afin de pouvoir énumérer certains défis auxquels la sécurité sociale mexicaine fait face en raison des catastrophes naturelles (II).

1 Les premières assurances sociales au monde étaient l'assurance maladie conçue par Otto von Bismarck, en Allemagne, en 1883. Elles avaient pour vocation de couvrir les travailleurs souffrant de maladies qui les empêchaient de travailler et donc de ne pas les priver de leur salaire. Voir dans Bundesministerium für Arbeit und Soziales, Bismarcks Sozialgesetze 1883-1889, Sozialgeschichte Infoblatt ein Arbeitsheft für die Schule, Berlin, 2013, p. 3 à 7. G. Mendizábal Bermúdez, La seguridad social en México, $2^{a}$ ed., Mexique, Porrúa, 2013, p. 72 et 73. 


\section{I - LE CADRE THÉORIQUE CONCEPTUEL}

Il convient ici de délimiter deux thèmes : les catastrophes socio-naturelles et ce qui les distingue des risques naturels, et l'évolution de la sécurité sociale comme droit humain, en tenant compte des principaux outils utilisés pour atteindre ces objectifs au Mexique, à savoir les assurances sociales², l'assistance sociale, etc., afin de pouvoir analyser le besoin de protection des victimes de catastrophes socio-naturelles et l'obligation de l'État de les protéger.

\section{A - LES CATASTROPHES SOCIO-NATURELLES}

L'une des principales causes du changement climatique est le réchauffement de la planète, qui entraîne une augmentation de la température de la surface de la terre, provoquant ainsi l'effet de serre. Cet effet se produit « lorsque certains gaz de lıatmosphère terrestre retiennent la chaleur. Ces gaz laissent passer la lumière mais gardent la chaleur comme les parois vitrées d'une serre ${ }^{3}$. " Les activités humaines qui accentuent l'effet de serre sont surtout la combustion de vastes étendues de végétation pour accroître la surface agricole ; lıutilisation massive de combustibles fossiles comme le pétrole, le charbon et le gaz naturel pour obtenir de liénergie ; les procédés industriels et les émissions de gaz provenant, notamment, des industries et des automobiles dont les conséquences sont notamment ${ }^{4}$ :

- L'augmentation ou la diminution des pluies sur presque toute la planète avec une augmentation de leur intensité.

- La diminution des glaciers de montagne et de la couverture neigeuse dans les deux hémisphères.

- Des sécheresses plus intenses et plus longues dans le monde entier.

- Des cyclones tropicaux plus violents.

- De nombreux systèmes biologiques et physiques sont affectés par les changements climatiques régionaux, notamment par la hausse ou la baisse des températures.

Les conséquences susmentionnées entraînent des catastrophes, dont les plus flagrantes sont les sécheresses, les inondations et les ouragans, généralement appelées catastrophes naturelles. Il convient toutefois de se demander s'il s'agit de catastrophes naturelles ou de catastrophes environnementales socio-naturelles.

2 Selon le professeur Néstor de Buen, «La sécurité sociale est en réalité une fin, et l'assurance sociale

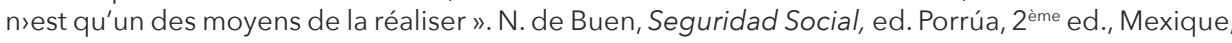
1999 , p. 16.

3 National Geografic, ¿Qué es el calentamiento global?, http://www.nationalgeographic.es/medio-ambiente/calentamiento-global/calentamiento-globaldefinicion,

4 A.R. Moreno Sánchez, et J. Urbina Soria, Impactos sociales del cambio climático en México, México, Instituto Nacional de Ecología (INE-SEMARNAT), 2008, p. 10. 
Le géographe américain Gilberto White ${ }^{5}$ a été le premier, dans les années 40 , à mener des études sociales sur les catastrophes naturelles, qui, depuis, ont bien évolué et font l'objet d'idées largement répandues:

1. Attribuer la cause des événements réels et quotidiens à un niveau suprahumain (divin), impossible à pénétrer, par exemple, une inondation est une punition de Dieu.

2. Un autre type de conception erronée et pernicieuse consiste aussi à attribuer les catastrophes au comportement et à l'action maléfique de la nature et de l'homme.

3. Les phénomènes naturels (compris comme une manifestation de la nature) qui provoquent des catastrophes sont cycliques et naturels et sont donc inévitables ;

Cependant, s'il existe des catastrophes d'origine naturelle, on fait aujourd'hui état de catastrophes d'origine non seulement naturelle, mais aussi humaine, c'est-à-dire que les hommes sont à l'origine de leur survenance directement par leurs actions ou indirectement par leurs défaillances, de sorte que nous en sommes tous responsables.

Ainsi, un risque naturel désigne la possibilité qu'un territoire et sa population soient affectés par un phénomène naturel d'un niveau extraordinaire ${ }^{6}$, dont la cause ou l'origine est inconnue. A ce propos, il convient d'attirer l'attention sur les aspects suivants :

a) Alors que les catastrophes naturelles n'ont d'autre responsable que les cycles de la nature, les désastres naturels causés par le changement climatique ont pour principale cause l'effet de serre (95\%), résultat de la pollution de l'environnement par l'homme, provoquant le réchauffement de la planète, qui, à son tour accentue des phénomènes climatiques comme les cyclones, la sécheresse, les inondations, la fonte des glaces des pôles et les vagues de froid ${ }^{7}$. L'abattage excessif et illégal d'arbres provoque, entre autres, l'érosion des sols et les rend propices aux incendies. La destruction des mangroves sur les côtes vient supprimer la protection naturelle contre l'érosion, les cyclones, les vents violents et les ouragans qui ont un impact direct sur les terres, les détruisant davantage ${ }^{8}$.

b) Les phénomènes naturels à l'origine des catastrophes sont inévitables. Par exemple, il est difficile de prévoir exactement quand et avec quelle ampleur un tremblement de terre va avoir lieu, bien que les zones les plus vulnérables aient été établies. En revanche, les catastrophes naturelles liées au changement climatique présentent des traits bien définis, comme les zones d'inondation par typhon, l'extension des zones désertiques, etc. Par conséquent, si les phénomènes naturels altérés sont inévitables, leurs conséquences, elles, sont évitables et contrôlables. Même si les spécialistes ont constaté que les dommages environnementaux sont irréversibles ${ }^{9}$ et ne peuvent

5 A. Maskrey, (comp.), Los desastres no son naturales, Red de Estudios Sociales en Prevención de Desastres en América Latina, 1993, p. 6, sur http://www.oei.es/decada/portadas/Desnat.pdf.

6 J. Ayala, Francisco, Riesgos naturales y desarrollo sostenible: impacto, predicción y mitigación, Ministerio de educación y ciencia, Madrid, 2006, p. 71.

7 Cf. AARP, 10 consecuencias del cambio climático, https://www.aarp.org/espanol/politica/historia/info-04-2013/fotos-cambio-climatico.html\#slide3.

8 FAO, La desaparición de manglares alcanza un nivel alarmante, http://www.fao.org/newsroom/es/news/2008/1000776/index.html.

9 IPCC, Fourth Assessment Report: Climate Change 2007, Synthesis Report, https://www.ipcc.ch/pdf/assessment-report/ar4/syr/ar4_syr.pdf. 
donc plus être évités à ce niveau, il convient d'enrayer leur développement et de mieux se préparer aux conséquences.

c) Une fois que l'on aura reconnu que les catastrophes sont dues aux actions ou aux négligences de la société, elles pourront être considérées comme des catastrophes socio-naturelles puisqu'elles sont la combinaison de phénomènes qui à l'origine n'étaient que naturels et de conséquences sociales. Ceci est essentiel dans le cadre de ce travail de recherche car ces catastrophes représentent alors un risque social.

\section{B - ÉVOLUTION HISTORIQUE DE LA PROTECTION CONTRE LES RISOUES : DES RISQUES LIÉS AU TRAVAIL AUX RISOUES SOCIO-NATURELS}

Il est nécessaire de comprendre les différentes étapes de l'évolution de la protection contre les risques dans les législations de tous les pays, et notamment la création des assurances sociales comme un outil de sécurité sociale efficace pour protéger les droits des assurés et de leurs ayant-droits. La protection qu'elles offrent marque un tournant dans l'évolution de la sécurité sociale, dans la mesure où elles établissent des obligations et des droits très clairs. Il convient de noter que, dans le cadre de ce processus de développement, on a doté les bénéficiaires des outils nécessaires pour faire valoir leurs droits aux prestations face aux besoins auxquels ils sont confrontés à des moments et à des endroits précis. Les risques n'ont pas toujours été pris en compte de la même manière, car de nouvelles situations nécessitant des protections apparaissent à mesure que la société évolue.

La société se doit de faire un très grand pas en matière de sécurité sociale : passer de la protection prévue par l'assurance des risques professionnels à la protection du risque psychosocial ou du risque socio-naturel couvert par la sécurité sociale. En effet, l'assurance sociale protège un groupe social défini, à savoir les salariés et leur famille au Mexique (moins de $60 \%$ de la population), alors que la sécurité sociale protège l'individu en toutes circonstances, tout au long de sa vie, par d'autres moyens, comme l'assistance sociale. Dans certains pays comme le Mexique, l'assurance sociale est l'instrument permettant aux travailleurs et à l'État d'unir leurs actions pour protéger le droit de la classe ouvrière à bénéficier de prestations (prestations en nature et financières qui les protègent de certains risques) liées à leur travail et permettre ainsi une meilleure qualité de vie. L'assurance sociale est un instrument de la sécurité sociale ; c'est la matérialisation ordonnée et systématisée de cette discipline juridique qui lui permet d'avoir des applications précises. Les principales théories de la protection contre les risques sont les suivantes ${ }^{10}$ :

\section{1 - Théorie de la faute civile ou pénale}

Cette théorie repose sur l'établissement de la faute, définie comme l'ensemble des conditions nécessaires à l'imputation subjective de l'acte à l'auteur ${ }^{11}$. Cette définition découle de l'obligation d'inclure dans une seule notion à la fois la faute et le dol. Cette théorie a été créée par le droit romain, qui retenait au travers de la notion de « diligence» lıobligation dıéviter tout préjudice par tous les moyens disponibles et via la notion de

10 Cf. G. Mendizábal Bermúdez, El acoso laboral y la seguridad social, México, Porrúa, 2013, p. 177.

11 Traduction personnelle à partir de G. Saccone, Lezione L'elemento sogettivo, Universitá Telemática, Roma, 2006, p. 7, http://www.unipegaso.it/materiali/PostLaurea/Saccone/Elemento_Soggettivo.pdf. 
«négligence » le fait de ne pas recourir à ces moyens; il y a donc faute lorsqu'un acte ou une omission affecte autrui.

Cette théorie s'appuie sur les doctrines pénales, notamment sur «la vision victimologique du dommage " selon laquelle "liauteur dıun dommage est tenu pour responsable ». Avec cette théorie, Ruiz Moreno renvoie au Code civil français et précise que «tout fait quelconque de l'homme, qui cause à autrui un dommage, oblige celui par la faute duquel il est arrivé à le réparer $»^{12}$. Ainsi, lorsqu'un risque se présente et qu'un dommage en découle, ce dernier doit être réparé par la partie coupable.

\section{2 - Théorie de la responsabilité contractuelle en droit civil}

Elle trouve son fondement dans l'existence d'un contrat préalable ; la responsabilité découle de la relation juridique; appliquée au contexte du travail, elle repose sur "l'obligation de l'employeur de veiller à la sécurité des travailleurs » ${ }^{13}$. Ainsi la faute ne sert plus à établir la responsabilité du risque créé et des dommages causés, la responsabilité découlant simplement du contrat de travail lui-même. C'est en ce sens que, lorsqu'un risque se présente, la personne responsable des conséquences sera celle désignée par le contrat.

\section{3 - Théorie du risque}

Cette théorie découle du machinisme et est adoptée dans les Codes civils. Elle trouve son origine dans l'impossibilité de rendre toute personne responsable lorsqu'un événement catastrophique ou préjudiciable n'est pas le fruit d'un acte délibéré, renvoyant ainsi la responsabilité sur la personne qui a généré le risque. C'est une théorie qui conduit à privatiser le risque. Dans cette logique appliquée au monde du travail, le propriétaire des machines ou des produits dangereux dans une industrie est responsable des dommages qui en résulteront. Cette théorie est aussi appelée théorie objective: est tenue pour responsable la personne chargée de surveiller l'objet dangereux, sans tenir compte de la notion de faute et de responsabilité contractuelle ${ }^{14}$.

\section{4 - Théorie du risque professionnel}

D'après cette théorie, lorsque l'employeur bénéficie des services de salariés, il devient responsable des aléas qu'ils subissent, comme l'ont souligné Alarcón et González : (...) «le système juridique attribue la responsabilité de la réparation des dommages causés sans faute ni négligence, mais simplement par la concomitance d'un facteur objectif préalablement déterminé qui, en cas d'accidents du travail, relève de la catégorie du « risque professionnel ». L'employeur est toujours responsable des risques créés par le seul fonctionnement de son entreprise ${ }^{15}$. Cette théorie est à la base de l'assurance sociale, il s'agit de la protection maximale accordée par certains pays à ce jour.

12 A.G. Ruiz Moreno, Nuevo derecho de la seguridad social, México, Porrúa, 1999, p. 483.

13 Idem.

14 Banco de Seguros del Estado, Accidentes del Trabajo y Enfermedades Profesionales -Edición Concurso 2010, p. 13 sur:

http://www.bse.com.uy/bse/archivos/accidentesdeltrabajoyenfermedadesprofesionales.pdf.

15 Cf. M.R. Alarcón Caracuel y S. González Ortega, Compendio de Seguridad Social, Ed. Tecnos, 4 ème éd. España, 1991, p. 20. 


\section{5 - La théorie du risque social}

Les risques auxquels tout homme dans la société est soumis sont appelés risques sociaux pour deux raisons selon Alarcón et González ${ }^{16}$ :

$1^{\circ}$ pour leur caractère général, puisqu'il s'agit de risques qui menacent tout un chacun. Il s'agit par exemple de la maladie ou des conséquences du changement climatique. A cela s'ajoute le fait que, dans de nombreux cas, les risques sont le produit d'une organisation sociale, par exemple la grève, ou sont inhérents à la vie en société, par exemple, une épidémie, un accident de voiture ou l'émission de gaz polluants, etc.

$2^{\circ}$ pour la conviction selon laquelle il incombe à la collectivité elle-même d'organiser la prévention et la réparation des conséquences néfastes ${ }^{17}$.

La sécurité sociale a ainsi évolué dans chaque pays en fonction des besoins de sa propre histoire ; mais son objectif principal reste le même : l'amélioration de la qualité de vie de tous les individus. En d'autres termes, la sécurité sociale représente la conscience organisée d'un peuple et doit protéger les individus sous la forme d'une assurance ou d'un moyen de protection sociale lorsque survient un risque social.

\section{6 - La théorie de la sécurité sociale comme droit de l'homme}

L'OIT définit la sécurité sociale comme la protection qu'une société offre aux personnes et aux ménages pour garantir l'accès aux soins de santé et à la sécurité du revenu, surtout en cas de vieillesse, chômage, maladie, invalidité, accident du travail, maternité ou disparition du soutien de famille ${ }^{18}$. Il est toutefois nécessaire d'analyser le concept de sécurité sociale à la lumière de la théorie des droits de l'homme, ce qui rend la définition précitée restrictive. C'est pourquoi il semble préférable de reconnaître la sécurité sociale comme un droit naturel inhérent à tout être humain, que l'État a l'obligation de garantir de manière systématique et pas seulement pour l'épanouissement de l'in dividu mais pour celui de la société en général ce qui fait que ce droit est reconnu par les systèmes juridiques en vigueur. En d'autres termes, la sécurité sociale ne se limite à aucun groupe social, au contraire, elle reconnaît que chaque individu, en tant que membre d'une société, a besoin d'être protégé contre tous les risques sociaux pour pouvoir se réaliser pleinement.

Comme on peut le voir, la définition de l'OIT repose sur la théorie du risque social qui s'est imposée dans certains pays, principalement européens et qui couvre toutes les personnes, qu'elles aient ou non une relation de travail formelle. Cela démontre à quel point il est nécessaire de protéger l'ensemble des groupes sociaux ou de la population des conséquences des différents risques (par exemple prise en charge des soins de santé des mères pendant la grossesse et l'allaitement, vaccin des enfants, soutien économique aux personnes âgées, prestations en nature pour soulager la dépendance physique des personnes âgées dans un pays comme le Mexique où la population est vieillissante).

16 Ibid.

17 Ibid., p. 16.

18 Organisation internationale du travail, Hechos concretos de la Seguridad Social, https://www.ilo.org/wcmsp5/groups/public/---dgreports/ 
Les assurances sociales sont les principaux outils d'une sécurité sociale fondée sur la théorie du risque social et permettent dıoffrir une protection sociale aux personnes touchées par les risques sociaux naturels.

Comprendre que la sécurité sociale est un droit de l'homme donne du pouvoir aux citoyens vis-à-vis de l'État comme en témoigne le néo-constitutionnalisme social latinoaméricain, les plus hautes juridictions de certains pays accordant sur ce fondement des prestations sociales indépendamment des exigences administratives établies par la législation, du simple fait de les considérer comme droits de l'homme.

\section{II - LES CONSÉOUENCES DES CATASTROPHES NATURELLES SUR LA POPULATION EN MATIĖRE DE PRESTATIONS DE SÉCURITÉ SOCIALE}

La qualité de vie des personnes dont l'environnement est frappé par les catastrophes socio-naturelles imputables au changement climatique se voit affectée de diverses manières. Ces conséquences portent atteinte au droit à la sécurité sociale de nombreux Mexicains, car elles se traduisent par des besoins qui doivent être couverts par la sécurité sociale, qui constituent à leur tour des droits: le droit à la santé, le droit à un logement décent et le droit à une sécurité des moyens de subsistance (toutes ces situations étant actuellement couvertes par la sécurité sociale). La plupart du temps, les prestations accordées ne répondent pas aux besoins et ne respectent pas les droits susmentionnés. Les principales conséquences des catastrophes socio-naturelles liées au changement climatique sur la qualité de vie de la population qui doivent être traitées par la sécurité sociale sont les suivantes:

a) Impact sur la santé publique. Outre les événements accidentels et soudains provoqués par les risques socio-naturels lors de leur survenance (tremblements de terre, cyclones tropicaux, inondations et éruptions volcaniques) tels que les traumatismes, les brûlures, les amputations, etc., le changement climatique affecte lentement mais continuellement la santé de toute la population, créant de graves problèmes de santé publique. A cet égard, on a constaté des signes d'augmentation excessive du rayonnement ultraviolet, cause des principaux cancers de la peau ${ }^{19}$; on a également fait état dıune augmentation des maladies infectieuses provoquées par la pollution des eaux et transmises par des animaux (comme les moustiques qui se propagent vers des zones géographiques où ils étaient auparavant inconnus) et de la propagation rapide des maladies contagieuses qui passent rapidement du stade de maladies locales au stade d'épidémies voire de pandémies comme l'Ebola, le $\mathrm{VIH}$, la grippe aviaire ; les allergies sont aussi un problème pour tout système de santé ; enfin, de nombreuses maladies respiratoires aiguës tuent chaque année les plus faibles, personnes âgées et enfants, en raison des conditions hivernales de plus en plus extrêmes ${ }^{20}$.

19 Voir Noticias de salud ambiental, Salud Pública de México, vol. 55, n 1, enero-febrero, 2013, p. 97. http://www.scielosp.org/pdf/spm/v55n1/v55n1a14.pd.

20 Voir R. Koch Institut und Umweltbundesamt, Klimawandel und Gesundheit, Allemagne, 2013, p. 7. http://www.rki.de/DE/Content/Kommissionen/UmweltKommission/Aktuelle_Informationen/ Downloads/klimawandel_gesundheit_handlungsempfehlungen_2013.pdf. 
b) Perte de revenus pour survivre. Les catastrophes entraînent la perte des moyens de subsistance de nombreux travailleurs qui se retrouvent alors sans emploi, ou dont la santé est affectée. C'est le cas des ouragans sur les côtes, qui dévastent les zones touristiques et laissent de nombreuses familles sans possibilité de se développer sur le plan économique.

c) Dégradation ou perte du logement. Au Mexique, le logement est considéré comme une prestation de sécurité sociale et il est fourni par l'assurance sociale (par l'intermédiaire de I'Instituto de Fondo Nacional para la Vivienda de los Trabajadores et du Fondo de la Vivienda del Instituto de Seguridad y Servicios Sociales de los Trabajadores del Estado). Les habitations sont gravement touchées par les catastrophes socio-naturelles, comme les ouragans, les inondations et les tremblements de terre. II est donc essentiel d'avoir une assurance pour protéger le patrimoine immobilier des travailleurs, que ce soit en cas de pertes totales ou partielles. Actuellement, seuls les logements donnant lieu à un crédit en cours bénéficient de cette protection. Les autres logements qui ne rentrent pas dans cette catégorie ne bénéficient d'aucun type d'assurance sociale et ne peuvent être couverts qu'à condition de souscrire à une assurance privée.

\section{A - La législation du Mexique face aux CATAStrophes naturelles}

Le Mexique a sa part de responsabilité dans le problème mondial du réchauffement climatique, puisqu'il émet $2 \%$ des gaz à effet de serre. Selon les données fournies par le Centre de recherche sur les écosystèmes de l'UNAM, le Mexique est le 14 ème émetteur de gaz à effet de serre au monde ${ }^{21}$. Le 12 décembre 2013, la Banque mondiale a publié un document intitulé : «Alianza Estratégica de País (AEP) 2014-2019 para los Estados Unidos Mexicanos ", visant à réduire l'extrême pauvreté et à accroître la prospérité pour tous et ainsi assurer un développement durable. Ce document indique que le Mexique émet 472 millions de tonnes de CO2 par an, soit environ 3,8 tonnes par habitant. Ces chiffres s'expliquent par l'utilisation et la production inefficaces de l'énergie et de l'industrie ${ }^{22}$. Les conséquences du changement climatique constituent un défi de taille : dégradation de l'environnement, appauvrissement de la biodiversité, pression urbaine croissante sur les ressources naturelles et, bien entendu, vulnérabilité des citoyens pauvres face aux catastrophes socio-naturelles.

Le pays est situé dans une zone géographique particulièrement vulnérable aux conséquences du changement climatique et donc aux catastrophes socio-naturelles. Le nord-ouest du territoire est affecté par les sécheresses et le sera de plus en plus, le sud-étant impacté par les inondations et les côtes étant de plus en plus touchées par les phénomènes hydrométéorologiques comme les ouragans et les tempêtes tropicales. À cela s'ajoutent les faiblesses des structures sociales et économiques. Si cette tendance se poursuit, les inégalités latentes s'accentueront dans de nombreuses franges de la population. Avec le

21 Agencia Iberoamericana para la Difusión de la Ciencia y tecnología, México ocupa el $14^{\circ}$ lugar del mundo en emisiones de gases de efecto invernadero, http://www. dicyt.com/noticias/mexicoocupa-el-14o-lugar-del-mundo-en-emisiones-de-gases-de-efecto-invernadero

22 Banque mondiale, Alianza Estratégica de País (AEP) 2014-2019 para los Estados Unidos Mexicanos, http://www.bancomundial.org/es/news/press-release/2013/12/12/WBG-New-CountryPartnership Strategy-for-Mexico-2014-2019. 
temps, les Mexicains ont compris que le changement climatique affecte l'économie et que de plus en plus de personnes sont victimes de catastrophes socio-naturelles parce qu'une grande partie de la population vit dans une pauvreté extrême. Par conséquent, une série de programmes et d'actions ont été mis en œuvre pour résoudre ce problème dont ceux évoqués par le SEMARNAT (Secrétariat à l'environnement et aux ressources naturelles du Mexique): Fiducie préventive (FIPREDEN); Fonds pour la prévention des catastrophes naturelles (FOPREDEN); Fonds national d'intervention en cas de catastrophe (FONDEN) ; Programme de prise en charge des zones de sécheresse récurrentes du SAGARPA (PIASRE); Programme d'emploi temporaire (PET) mis en œuvre principalement par le SEDESOL.

Pour la première fois, le Plan national de développement (PND) 2007-2012 fait du changement climatique un thème prioritaire de la planification du développement du pays. L'objectif principal est d'encourager des mesures d'adaptation aux effets négatifs du changement climatique ; ces objectifs ont été intégrés depuis cette année dans le plan de développement. Entre 2013 et 2018, certains objectifs ont été mis en œuvre dans l'Accord de Cancun, à savoir :

- le Programme spécial sur le changement climatique 2013-2018 (PECC). Le programme a présenté 142 objectifs d'adaptation, dont $46 \%$ concernent les instruments de planification, $28 \%$ les études et publications, $20 \%$ sont des objectifs concrets ayant un impact direct sur la société et le territoire et $6 \%$ sont des actions de renforcement institutionnel, avec trois volets essentiels : -2008-2012 : l'évaluation de la vulnérabilité et l'évaluation économique du pays ; 2013-2030 : le renforcement des capacités stratégiques d'adaptation, et 2030-2050 : la consolidation des capacités renforcées ${ }^{23}$.

\section{B - MESURES SPÉCIFIQUes de SÉCURITÉ SOCIALE CONTRE LES RISQUES SOCIO-NATURELS}

Si nous ne bénéficions pas de mesures ou de progrès notables, comme c'est le cas des Japonais ${ }^{24}$, pour faire face aux conséquences des catastrophes naturelles, nous disposons de normes juridiques qui attestent que les assurances sociales ont pris la mesure du problème et qui peuvent servir de base pour étendre la protection nécessaire. La législation mexicaine en matière de sécurité sociale, et en particulier celle sur les principales assurances sociales (IMSS et ISSSTE) comprennent des dispositions spécifiquement applicables à la prise en charge des victimes d'une catastrophe socio-naturelle. Il s'agit de la Loi sur la sécurité sociale. Prestations de solidarité sociale: Section IV. Des prestations de solidarité sociale

23 Ibid., p. 85 à 89.

24 Par exemple, ils ont un programme intitulé «Assistance pour le rétablissement du mode de vie des victimes", qui prévoit une assistance aux victimes pour leur permettre de retrouver leur mode de vie auto suffisant. Parmi les aides offertes figurent de l'argent pour des condoléances en cas de catastrophe, pour les personnes handicapées, pour accompagner la reconstruction personnelle des victimes de catastrophes, et des prêts sous forme de fonds d'aide en cas de catastrophe et de fonds d'assistance sociale. Dirección General de Administración de Desastres, Administración de desastres en Japón, Oficina de Gabinete, p. 4,

http://www.ve.embjapan.go.jp/esp/image/ADMINISTRACION\%20DE\%20DESASTRES\%20 EN\%20JAPON.pdf 
Article 214. Les prestations ou services de solidarité sociale comprennent les soins de santé communautaires, médicaux, pharmaceutiques et le cas échéant hospitaliers, sous la forme et dans les conditions prévues aux articles 215 à 217 de cette loi.

Article 216 A. L'Institut devra prendre en charge les personnes non bénéficiaires dans les cas suivants : I. En cas d'urgence nationale, régionale ou locale ou en cas de catastrophe naturelle; II. Dans le cas de campagnes de vaccination, de soins de santé ou de promotion de la santé, et III. Afin de soutenir les programmes de lutte contre la marginalisation et la pauvreté, lorsque l'Exécutif fédéral l'exige.

Aux fins du point l, l'Exécutif fédéral, par l'intermédiaire du Ministère des finances et du crédit public, fournit à l'Institut les ressources financières correspondantes conformément aux dispositions applicables... Le dernier article reconnaît à la fois l'existence du problème des catastrophes naturelles au Mexique et le droit aux soins médicaux de la population en général, sous réserve d'être victime d'une catastrophe naturelle, indépendamment du lien juridique d'assurance. L'article 4 de la loi de l'Institut de sécurité et des services sociaux pour les agents de l'État prévoit le droit d'obtenir un prêt personnel pour les assurés en cas de catastrophe naturelle et les paragraphes 162, 239 et 240 prévoient le montant et les conditions d'éligibilité : Article 4. Les prestations et services suivants sont obligatoires : II. Prêts personnels : a) Ordinaires; b) Spéciaux; c) Pour l'acquisition de biens de consommation durables, et d) Extraordinaires pour les victimes de catastrophes naturelles; ...

Article 162. Les prêts personnels sontaccordés aux travailleurs et retraités conformément au programme annuel autorisé par le Conseil d'administration de l'Institut, en fonction de la solvabilité du Fonds lui-même et conformément à ce qui suit : Il. Les prêts sont accordés en fonction des capacités financières du Fonds et conformément aux règles établies par le Conseil d'administration de l'Institut, et se déclinent en quatre catégories, à savoir : d) (Prêts) Extraordinaires pour les victimes de catastrophes naturelles. Le montant est fixé par le Conseil d'administration de l'Institut;

Article 239. L'Institut constitue la Réserve opérationnelle pour des imprévus et le financement visés au présent chapitre, selon trois enveloppes : prévisionnelle, catastrophe et spéciale : Il. L'enveloppe catastrophe peut être utilisée pour couvrir des frais de tout type pour faire face à des catastrophes naturelles ou à des cas de force majeure qui, de par leur nature, n'ont pas été assurés.

Article 240. La Réserve opérationnelle pour imprévus et financement est constituée, alimentée ou reconstituée trimestriellement jusqu'à concurrence d'un montant équivalent à soixante jours du revenu total de l'Institut l'année précédente, sans tenir compte des sommes relatives à l'assurance retraite, à la pension retraite et au Fonds de logement.

....L'Institut, avec l'autorisation préalable du Conseil d'administration, peut disposer des ressources affectées à la Réserve de fonctionnement pour imprévus et financement, pour couvrir tout imprévu jusqu'à concurrence d'un montant équivalent à quatre-vingt-dix jours du revenu moyen de l'année précédente de l'assurance ou du service pour lequel ce financement a été demandé...

Comme on peut le constater, la réglementation n'est pas suffisante pour répondre aux besoins réels, ce qui exige de procéder à des ajustements aussi bien juridiques que financiers pour faire face aux conséquences des catastrophes naturelles et socio-naturelles. Il convient donc de souligner qu'outre le respect de la législation nationale en cas de risques socio-naturels, les directives internationales axées sur la protection en cas de catastrophe 
doivent aussi être respectées. C'est le cas par exemple de la Recommandation ( ${ }^{\circ} 205$ ) de l'OIT sur l'emploi et le travail décent pour la paix et la résilience, 2017, qui, bien qu'elle n'inclut pas spécifiquement les victimes de certains risques socio-naturels, établit les mesures à prendre, en cas de catastrophe, en faveur de l'emploi et du travail décent pour la prévention, le redressement, la paix et la résilience face aux situations de crise résultant de conflits et de catastrophes ${ }^{25}$.

Les stratégies exposées dans la Recommandation 205 visent entièrement à protéger les travailleurs en fonction de leur source de revenus, la première stratégie étant l'introduction de mesures immédiates pour l'emploi et la protection sociale, et la seconde la promotion de la récupération des sources d'emploi afin de créer des emplois décents et durables. II convient de noter que le Mexique n'a pas suivi cette Recommandation. Néanmoins, c'est une Recommandation qui prépare sans aucun doute le terrain pour étendre la protection en cas de risques socio-naturels.

\section{C - LES DÉFIS DE LA SÉCURITÉ SOCIALE MEXICAINE}

Malgré les grandes transformations que connaît le monde, y compris l'évolution du concept même de sécurité sociale, son objectif reste toujours le même: améliorer la qualité de vie de chaque individu dans la société. Autrement dit, la sécurité sociale demeure la conscience organisée d'un peuple et doit protéger les individus en leur offrant une assurance ou un moyen de protection sociale lorsque survient un risque social. C'est en ce sens que le premier défi de la sécurité sociale, et le plus important, est de reconnaître que les conséquences des catastrophes naturelles menacent tout un chacun, qu'elles sont le résultat de l'organisation sociale et qu'il faut donc bien comprendre qu'il incombe à la collectivité elle-même d'organiser la prévention et de participer à la réparation des conséquences dévastatrices. Ceci étant dit, les défis suivants peuvent alors être identifiés:

1 - Reconnaître l'importance du rôle des assurances en cas de catastrophes naturelles, en particulier celui des assurances sociales. Si de nombreux pays ont commencé à s'assurer contre les dépenses considérables occasionnées par les catastrophes socio-naturelles, cette assurance a été limitée à l'assurance commerciale et doit être étendue à l'assurance sociale qui, en tant qu'instrument principal et le plus efficace de la sécurité sociale, a démontré, en temps de crise ou non, qu'elle est en mesure de fournir les services sociaux nécessaires auxquels aucun autre mécanisme de protection sociale ne peut parvenir : ce fut le cas par exemple de l'assurance chômage offerte en Europe lors de la crise financière de 2009, des soins de santé pour l'ensemble de la population lors des événements sanitaires qui ont touché le Mexique avec la grippe H1N1, des moyens de confinement et de réparation massive au Japon après le tremblement de terre de 2011.

2 - Mieux vaut prévenir que guérir. La sécurité sociale doit être préventive et proactive. Depuis le lancement de la vision de l'AISS d'une "Sécurité sociale dynamique » en 2007, des changements importants ont été signalés par lıorganisme lui-même: "Les systèmes

25 OIT, Recomendación sobre el empleo y el trabajo decente para la paz y la resiliencia, $2017\left(n^{\circ} 205\right)$. 
de sécurité sociale ont fait diénormes progrès dans le renforcement de leur rôle protecteur, proactifet préventifet sont devenus plus accessibles $»^{26}$. Les actions menées par ces systèmes de manière préventive et proactive pour réduire les conséquences des catastrophes causées par le changement climatique ne font pas l'objet de véritables analyses.

Les risques sociaux causés par les catastrophes socio-naturelles entraîneront une augmentation des dépenses de sécurité sociale et ce principalement en raison de l'émergence de nouvelles pandémies et de nouveaux besoins nutritionnels (du fait de la sécheresse, de l'apparition de nouveaux groupes vulnérables : la population la plus exposée est très sensible et/ou dispose d'une faible capacité d'adaptation, c'est le cas principalement des pauvres, des enfants et des personnes âgées).

Il est nécessaire de voir dans la sécurité sociale un mécanisme de redistribution des richesses. On peut souligner qu'au cours des dernières décennies, la sécurité sociale a perdu son rôle prédominant de mécanisme de redistribution des richesses, du fait qu'au Mexique, du moins, elle n'a pu élargir sa protection par le biais des assurances sociales et s'est limitée à la protection par le biais de l'assistance sociale. Par conséquent, les personnes vivant dans la pauvreté ne bénéficient pas d'un mécanisme efficace de répartition des dépenses entre les membres de la société. En plus de cela, dans une société, les personnes les plus touchées par les conséquences des catastrophes socio-naturelles sont précisément les plus pauvres, puisqu'elles vivent dans des zones marginales fortement exposées aux dégâts (près des rivières, des ravins et des lacs, qui, non seulement, sont pollués, mais sont aussi sujets aux inondations) où les services de santé sont insuffisants en raison de lıéloignement des communautés. En outre, leur état nutritionnel est mauvais et les sécheresses entraîneront des famines qui les affecteront plus directement en raison du prix élevé de la nourriture ; les maladies qui ont disparu, ou presque, augmenteront comme la malaria, la fièvre typhoïde, et d'autres; les vagues de froid et de chaleur vont s'intensifier et durer de plus en plus longtemps, et provoqueront vraisemblablement la mort des personnes qui pourront le moins bien les affronter, à savoir les plus démunis. En résumé, la pauvreté est un facteur qui empêche, ou du moins entrave, le processus d'adaptation au changement climatique et les citoyens en situation de pauvreté ont donc besoin de la solidarité de la sécurité sociale pour faire face aux conséquences.

3 - Identifier de nouvelles formes de financement. Tout comme la sécurité sociale doit s'adapter aux nouveaux risques sociaux auxquels elle est confrontée aujourd'hui, par exemple en couvrant les risques sociaux liés au réchauffement climatique, il est nécessaire d'établir de nouveaux mécanismes de financement de la sécurité sociale, qui incluent non seulement des contributions à de nouvelles prestations couvrant ce risque social, mais également des mécanismes de financement indirects.

Apporter de nouveaux avantages économiques et en nature. Ces derniers doivent avoir comme objectif d'en atténuer les conséquences (par exemple, des prêts en cas de catastrophes naturelles, des allocations de chômage, des pensions aux orphelins des victimes de catastrophes socio-naturelles, etc.) mais surtout de les prévenir, grâce à la médecine préventive, par des contributions visant à couvrir les dépenses liées aux catastrophes imputables au réchauffement climatique, etc.

26 H.H. Konkolewsky, (ISSA Secretary General), Developments and Trends in Social Security, www.issa.int/wssf2013. 


\section{SÉCURITÉ SOCIALE ET CATASTROPHES SOCIO-NATURELLES}

Enfin, il reste aussi à trouver des solutions aux défis à long terme: la couverture universelle, qui exige de se pencher sur la nécessité de désolidariser la sécurité sociale du monde du travail, sans quoi on ne peut parler de protection des populations sans assurance et encore moins des risques découlant des catastrophes naturelles.

\section{Conclusions}

Si les catastrophes naturelles touchent des personnes dans le monde entier, celles causées en partie par le comportement de l'homme ont des conséquences encore plus graves sur la santé des personnes, la perte des sources de revenus ou la disparition du soutien de famille, etc. Autrement dit, les catastrophes socio-naturelles produisent des risques qui, lorsqu'ils se matérialisent, deviennent des besoins pour la population et doivent être reconnus comme des risques sociaux (générés par l'intervention de l'homme). Il incombe donc à l'État d'y répondre de manière rapide, adéquate et exhaustive en assurant la prévention et la réparation des conséquences sur la société.

Ces risques sont causés par lıorganisation sociale; il faut donc reconnaître qu'il revient à la collectivité elle-même d'organiser la prévention ainsi que la réparation des conséquences. Par conséquent, les assurances sociales, en tant que principal outil de la sécurité sociale, ne protègent plus seulement les individus contre les risques liés au travail, mais étendent leur couverture aux risques sociaux, c'est pourquoi la protection des risques causés par les catastrophes socio-naturelles est une question qu'il faut commencer à appréhender de manière concrète.

\section{GABRIELA MENDIZÁBAL BERMÚDEZ}

Professeur chercheur, Faculté de droit et de sciences sociales de l'Université autonome de l'État de Morelos

Thèmes de recherche : Industrie 4.0 et sécurité sociale, droit international de la sécurité sociale, sécurité sociale pour les groupes vulnérables.

\section{Publications:}

G. Mendizábal Bermúdez, (Coord.) Industria 4.0, Trabajo y Seguridad Social, Instituto de investigaciones jurídicas, UNAM, México, 2019.

G. Mendizábal Bermúdez, "¿Nuevo modelo de seguridad social en el contexto de la industria 4.0?", Revista Internacional y Comparada de Relaciones Laborales y Derecho del Empleo, ADAPT, Italia, 2018.

G. Mendizábal Bermúdez, Labour Inspektion, Zeitschrift für ausländisches und internationales Arbeits- und Sozialrecht, 2016, Alemania, 2018. 